\title{
Reflets
}

Revue ontaroise d'intervention sociale et communautaire

\section{Entrevue avec Caroline Andrew}

\section{Nérée St-Amand}

Volume 6, numéro 2, automne 2000

Problèmes sociaux en Ontario français

URI : https://id.erudit.org/iderudit/026314ar

DOI : https://doi.org/10.7202/026314ar

Aller au sommaire du numéro

Éditeur(s)

Reflets : Revue ontaroise d'intervention sociale et communautaire

ISSN

1203-4576 (imprimé)

1712-8498 (numérique)

Découvrir la revue

Citer ce document

St-Amand, N. (2000). Entrevue avec Caroline Andrew. Reflets, 6(2), 20-31.

https://doi.org/10.7202/026314ar

Tous droits réservés (C) Reflets : Revue ontaroise d'intervention sociale et communautaire, 2000

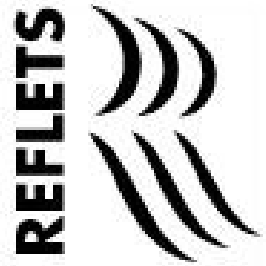

Ce document est protégé par la loi sur le droit d'auteur. L'utilisation des services d'Érudit (y compris la reproduction) est assujettie à sa politique d'utilisation que vous pouvez consulter en ligne.

https://apropos.erudit.org/fr/usagers/politique-dutilisation/ 


\section{Entrevue avec Caroline Andrew}

Caroline Andrew est doyenne, Faculté des sciences sociales, Université d'Ottawa. Nérée St-Amand l'a rencontrée, en juillet dernier, pour discuter des problèmes sociaux en Ontario français.

Reflets: Pour ce douzième numéro de Reflets, nous avons pensé traiter des problèmes sociaux en Ontario français. Étant donné l'évolution de la conjoncture sociale, politique et économique de la province, et particulièrement dans le contexte franco-ontarien, nous désirons explorer et analyser quelques grands problèmes sociaux de l'heure. C'est le défi que nous nous sommes proposé de relever.

Fernand Dumont publiait, en 1994, un Traité des problèmes sociaux. Ce classique analyse certains problèmes contemporains dans les domaines de la santé, des valeurs de société, de la participation et de l'exclusion, des inégalités économiques et sociales. Les derniers chapitres nous invitent à explorer les solidarités communautaires. En tant que personne très engagée - vous êtes grandement impliquée dans le social, c'est connu et reconnu - comment voyez-vous les problèmes sociaux évoluer, présentement, en Ontario?

C.Andrew : Je pense que c'est un très bon thème pour Reflets parce que nous sommes témoins de l'éclosion de plusieurs problèmes sociaux, qui sont des phénomènes difficilement analysables si nous nous en tenons à un unique facteur causal. De fait, plusieurs facteurs sont en cause, et ceux-ci sont reliés à l'impact de la mondialisation sur les individus, qui se fait davantage sentir dans les moyennes et les grandes villes. Les propos sur le thème de l'exclusion dans le livre de Dumont rendent compte en partie de ce que nous voyons comme les grands problèmes sociaux de l'heure. La problématique des sans-abri est une des manifestations les plus visibles de l'accélération des problèmes sociaux dans un pays comme le nôtre, 
où un tel phénomène ne devrait pas exister. Il s'agit d'un problème complexe parce que le fédéral et les provinces ont abandonné le logement social; il est davantage accentué par des politiques de désinstitutionnalisation auxquelles ne sont pas rattachées les ressources nécessaires. Les problèmes de toxicomanie s'ajoutent à des questions de pauvreté, à des questions de violence, particulièrement contre les femmes. La question des sans-abri exige donc une intervention plus complexe, multiforme, pour s'attaquer aux causes du problème qui est issu de toute une série de facteurs, dont l'abandon par les gouvernements de leurs responsabilités, tant au niveau fédéral que provincial.

De plus, le discours prônant le retrait de l'État-providence est tellement présent : les médias favorisent évidemment le marché privé qui, selon eux, va résoudre tous nos problèmes, tant économiques que sociaux. Ils s'évertuent aussi à culpabiliser les gens qui ne réussissent pas. Cela fait en sorte que les personnes exclues n'ont présentement plus de forum pour s'exprimer. Elles ont été grandement affectées par les coupures, du gouvernement provincial par exemple, et de plus, elles ont honte de leur situation. Les audiences populaires sur la pauvreté à Ottawa, il y a 2 ans, l'ont clairement démontré : des gens venaient s'excuser parce qu'ils n'avaient pas pu réussir alors qu'ils étaient confrontés à des circonstances impossibles causées par de grandes maladies, par des dépenses inattendues, ou par le manque d'emplois.

Pour revenir aux sans-abri, le problème est accentué par une sérieuse pénurie de logements; dans un tel contexte, les propriétaires sont enclins à mettre rapidement les gens à la porte s'ils ne payent pas leur loyer. Donc, il y a toute une série de phénomènes, de problèmes très complexes, qui s'expliquent, d'une part, par la tendance du gouvernement à vouloir responsabiliser les individus et, d'autre part, par le fait que les individus ou les communautés n'ont pas les moyens de se prendre en charge.

Enfin, beaucoup des problèmes sont accentués par la polarisation accrue entre riches et pauvres. Notre société regroupe, d'un côté des pauvres sans ressources et de l'autre, des gens vivant dans l'abondance. Qui plus est, les politiques économiques ont tendance à éliminer le communautaire, contribuant ainsi à amplifier la 
situation de marginalisation extrême d'une part et d'abondance de l'autre.

Reflets : Vous avez déjà mentionné quelques causes des problèmes sociaux actuelsla dévolution et le désengagement de l'État, toute la dimension idéologique et politique véhiculée par les médias en faveur des pratiques et de l'idéologie néolibérales. Quels seraient d'autres facteurs importants qui contribueraient à complexifier les problèmes sociaux d'aujourd'hui?

C.Andrew: Le fait que nos communautés regroupent des gens venus de nombreux pays pour s'établir au Canada et que les gouvernements se désistent face à plusieurs problématiques qu'ils renvoient à des réseaux communautaires de plus en plus hétérogènes ajoute encore à la complexité des enjeux actuels.

Reflets : Comment expliquer qu'une population hétérogène complexifie certains enjeux?

C.Andrew : Il n'y a pas qu'une façon d'inclure les réalités culturelles. Il faut constamment questionner le type d'intervention communautaire proposé, en essayant de se baser sur une analyse fondée sur l'ensemble de cette problématique. Cela ajoute à la complexité du problème parce que, dans les périodes difficiles comme maintenant, certains groupes ou individus sont davantage portés à blâmer d'autres secteurs plus défavorisés de la population. De nos jours, il faut beaucoup plus d'imagination pour tenter de faire valoir les retombées positives de l'interculturalité. Ici comme ailleurs, on privilégie des réponses faciles même si les problèmes sont complexes. Il ne faut pas non plus tomber dans le piège de dire au sujet des personnes immigrées : "Pauvres eux autres! C'est tellement difficile...» Par ailleurs, il faut reconnaître que c'est un enjeu relativement récent. Pour répondre à ce nouveau défi, le gouvernement Harris nous renvoie à une période glorieuse où les familles étaient merveilleuses et où, nous, les femmes, faisions de la bonne soupe et tout ça. Dans la période actuelle de grande diversité culturelle, cette tendance de retour vers une sorte de passé imaginaire reste facile, invitante même.

Reflets : Vous avez parlé un peu de la situation actuelle au Canada, en Ontario. Comment, d'après vous, semble se dessiner le futur? Comment évoluent actuellement les problèmes sociaux? 
C.Andrew : D'abord, parce que je suis une personne optimiste et aussi parce qu'il est important de garder une vision de l'histoire qui n'est pas construite d'avance, je reste convaincue que nous avons des options. Nous voyons actuellement plusieurs exemples où les gens sont conscients des problèmes, de l'évolution sociale. Par exemple, lors d'une conférence sur les technopoles, il y quelques années, des représentants des Chambres de commerce de grandes villes américaines de haute technologie avouaient qu'il y a des limites à tant miser sur un développement polarisé et que, à un moment donné, même le développement des secteurs sûrs s'en trouve menacé.

Même dans l'idéologie néolibérale, il y a donc des courants qui se confrontent, sur des questions de sécurité par exemple. En même temps, par contre, notre société se dirige de plus en plus vers la polarisation, démontrée par le fait que de nombreuses personnes deviennent moins généreuses et moins tolérantes envers les programmes de redistribution du revenu et qu'elles clament haut et fort : "La priorité c'est de mettre plus d'argent dans mes poches ».

Reflets: Donc, une décroissance de la conscience sociale des citoyens?

C.Andrew: Oui, et une philosophie qui veut que «le plus important, c'est moi,je peux me débrouiller seul ». Donc, on se désolidarise. C'est trop facile d'agir ainsi, dans une société où les gens relativement à l'aise ont peu de contact avec les pauvres. Donc, on peut imaginer une société davantage polarisée dans l'avenir. Mais ce scénario n'est pas inévitable; il y a un travail important à faire afin d'empêcher que cela ne se produise.

Il faut dénoncer la polarisation à cause de son impact sur ceux qui sont exclus. Mais il est aussi important de dire qu'éventuellement ça va faire mal même à ceux qui sont prospères parce qu'une trop grande polarisation crée une société instable. C'est l'assèchement du potentiel de tout un secteur de personnes exclues par les lois du marché.

Le discours sur la pauvreté des enfants, par exemple, est tendancieux parce qu'il cache de grandes contradictions. Il y a néanmoins des choses importantes dans ce discours qui nous alertent aux conséquences à long terme de ne pas remédier 
immédiatement à la pauvreté des enfants. Brimer l'imagination d'un enfant vivant dans la pauvreté affecte un être humain très profondément, et pour la vie. Il est important de faire prendre conscience de ces enjeux à long terme tout en reconnaissant l'ambiguïté du discours.

Reflets: Les solutions qu'on semble proposer, généralement, à la pauvreté des enfants— pour prendre cet exemple-là — sont souvent très limitées, ou très bornées, si on peut dire. Elles se limitent à donner un peu de nourriture ou quelques vêtements. Au lieu de la percevoir comme un problème social global, on traite la situation comme une urgence, sans plus.

C.Andrew : C'est d'ailleurs ce qu'on fait avec beaucoup de problèmes sociaux. On tente d'appliquer des pansements de dernière minute, plutôt que de réfléchir plus en profondeur à la redistribution des ressources.

Reflets: Si on parle de l'Ontario, en particulier, comment évaluez-vous le gouvernement en place? Comment voyez-vous les interventions $d u$ gouvernement Harris dans le contexte actuel d'une pauvreté de plus en plus accentuée?

C.Andrew: Évidemment, il y a toutes sortes de manières d'analyser les interventions gouvernementales. On peut dire que les gens qui appuient les politiques de la droite ont d'abord eu recours à des attaques contre les pauvres de façon très électoraliste-peut-être plus électoraliste qu'idéologique dans un certain sens. Il s'agit d'une tactique déplorable, dangereuse. On peut voir à quel point c'est dangereux quand les gens qui appuient le parti conservateur pour ces raisons-là jugent que les politiciens agissent de façon responsable en faisant la chasse aux pauvres. Le gouvernement a pris certaines mesures fracassantes, comme diminuer le niveau de l'assistance sociale et terroriser les pauvres en mettant en place des mécanismes de délation. Quant aux mesures prises contre les squeegees, elles sont aussi très électoralistes. Mais il s'agit d'abord d'une vision inquiétante de la vie en société.

Un livre publié récemment, Lost in the Suburbs, décrit la grande zone autour de Toronto. Les gens qui demeurent dans ces banlieues, loin du centre urbain, se sont endettés en achetant des propriétés et travaillent comme des fourmis pour parvenir à payer leurs 
maisons. Mais ils sont devenus les nouvelles victimes d'un individualisme agressif, dans le sens qu'ils veulent des réductions de taxe pour pouvoir payer leur factures. Ils proposent une sorte de marché privé de toutes les ressources collectives. Mike Harris est très lié à ce type d'électorat. C'est d'ailleurs un politicien qui attache une grande importance à l'image. Je trouve qu'il consacre beaucoup d'effort pour paraitre capable de prendre de dures décisions lorsqu'il coupe des programmes ou encore lorsqu'il propose la fusion de villes, comme c'est le cas à Ottawa. On commence tout juste à voir les conséquences de la fusion de Toronto. Cette décision, en particulier, n'a pas du tout entraîné les épargnes escomptées.

Cette fusion démontre, d'ailleurs, la façon d'agir de ce gouvernement : donner l'impression d'être bon gestionnaire d'état, prêt à prendre des décisions difficiles. Ce gouvernement est très préoccupé par l'aspect des relations publiques. D'où l'importance de projeter l'image de pouvoir dire des choses effrayantes parfois. Il y a certainement eu des mesures répressives importantes, mais son discours fait aussi partie de la stratégie très populiste, très électoraliste qu'il utilise.

Reflets: Je ne sais pas si vous avez reçu dans la poste, récemment, un dépliant qui illustre les résultats des réformes du gouvernement conservateur ontarien. C'est très éloquent, si on peut dire. Dans un sens, c'est très populiste, simpliste également. On y décrit les orientations, les prises de décision puis les résultats des politiques du gouvernement actuel. Il semble être en train de préparer la prochaine élection en parlant $d u$ workfare. Comme vous l'avez dit au tout début, c'est sur le dos de certaines catégories de gens vulnérables que les élections se font : les assistés sociaux, les femmes, chefs de familles monoparentales, etc.

C.Andrew : Oui, dans ce document-là, un certain thème revient constamment : celui de la fraude qu'on prétend avoir éliminée. Lorsque ce gouvernement parle du workfare, il inclut toujours un deuxième message qui encourage les gens à dénoncer leurs voisins. Il y a eu d'abord cette idée qu'il faut aider les gens à pouvoir travailler, mais il y a également une série de messages beaucoup plus punitifs et contrôlants. En même temps, il faut avouer que c'est habilement présenté... 
Reflets: Quand on pense à cette polarisation dont vous avez parlé tout à l'heure, puis à l'évolution des problèmes sociaux, y a-t-il des pistes de solutions plus pertinentes que d'autres dans ce que vous entrevoyez, dans ce qui se pratique, ou se discute actuellement?

C.Andrew : Certainement. Malgré les ambiguiités qu'engendre le retour au communautaire et au bénévolat, il y a de bons aspects à ce mouvement, parce qu'un des éléments qui est lié aux problèmes sociaux actuels, c'est une sorte de sentiment de déshumanisation du social. Le recours à une action très directe et locale me semble une façon d'essayer de reconnaitre la dignité humaine. Mais il faut être prudent parce que c'est aussi une sorte de désengagement qui libère le gouvernement, qui l'excuse en disant que le secteur privé devrait agir. Or, le secteur privé, on le sait, propose toutes sortes de pratiques et de politiques dont certaines n'ont pas été aussi progressistes!

Reflets: Donc, vous voyez un potentiel intéressant dans le développement local qui a été forcé de prendre la relève suite au désengagement de l'État?

C.Andrew : Je vois quelque chose de très intéressant mais en même temps de très inquiétant. Intéressant dans le sens ou ça peut correspondre à un effort visant à tisser une société civile qui a une certaine vitalité. Il faut cependant que l'État reste présent, qu'il continue de jouer un rôle. L'État doit maintenir des structures pour faciliter l'action collective pour les personnes défavorisées. Cet équilibre est important. Mais l'idée d'une certaine renaissance du local me semble aussi une des voies prometteuses.

Reflets : "Renaissance du local ", quelle expression intéressante! Mais comment fait-on pour concilier cela avec un rôle essentiel de l'État? Comme vous le suggérez, on ne veut tout de même pas que l'État abandonne ses responsabilités au secteur communautaire ou alternatif ou local. Quel équilibre voyez-vous entre ces deux forces, qui pourraient être complémentaires, mais qui pourraient aussi adopter des stratégies très différentes, voire opposées?

C.Andrew : Il est important de concevoir un système au sein duquel des balises seraient établies de telle sorte qu'une certaine redistribution des ressources relèverait du gouvernement central. Plutôt que de contrôler le local, le gouvernement doit viser à le libérer. Mais, 
en même temps, il faut un certain mécanisme pour prévenir une nouvelle forme d'oppression. La société civile qui émergera pourra alors être une force progressiste, alors que, il y a vingt ans, on se lamentait que l'intervention étatique était un étau qui brimait les gens. Il faut, par ailleurs, une grande vigilance de la part de citoyens et des procédures claires pour permettre au local d'opérer. Cela doit se faire à l'intérieur de normes générales et globales.

Reflets: Donc, les grandes lignes directrices devraient venir de l'État central, si je vous comprends?

C.Andrew : Certainement, nous avons besoin de modalités de redistribution. Par contre, il me semble plus difficile de suggérer quelle forme devraient prendre, par exemple, des normes nationales. Quels moyens, quelles marges doit-on permettre? Comment peut-on s'accorder une certaine mouvance de la base avec une gestion nationale des problèmes sociaux?

Reflets: Qu'est-ce qui arrive s'il y a des forces de conscientisation qui éveillent les populations réprimées? Je pense, par exemple, au logement à Toronto ou aux ressources alternatives en psychiatrie. Ce sont aussi des mouvements de la base. S'il y a des réseaux de psychiatrisés qui se prennent en charge et qui disent non à la psychiatrie, par exemple, qui disent non à un logement de troisième classe, qui disent "on a des droits " et qui les revendiquent. Ces groupes communautaires sont rapidement réprimés actuellement. Ce sont des mouvements qui naissent et qui pourraient être conscientisants. Mais l'État n'a pas un rôle neutre là-dedans...

C.Andrew : Non, justement, la scène politique présente toutes sortes d'exemples de différents mouvements qui visent la mobilisation collective. Je reviens à l'exemple des sans-abri. Il serait essentiel de créer des espaces de débats dans notre société, pour apporter un plus grand éclairage sur certaines questions et pour avoir plus d'échanges sur les causes des problèmes sociaux de l'heure.

En lisant les journaux, on perçoit une sorte de malaise de la civilisation, mais qui finalement est analysé d'une façon très simpliste. Il y a même des tendances scientifiques et académiques qui proposent que tout s'explique par des facteurs génétiques. Je pense qu'il faut un espace où débattre ces grands problèmes. Il se peut que ces travaux scientifiques aient un certain fondement. 
Mais il faut se rappeler qu'un des grands facteurs de pauvreté demeure la dimension politique. Les journaux offrent presque l'impression d'une sorte de vision sans possibilité de débat sur cette question de la génétique. On observe une sorte de retour à des facteurs individuels, psychologiques pour expliquer les problèmes sociaux. Il s'agit d'une vision de l'individu, mais qui a des implications importantes sur les formes d'intervention qu'on veut proposer par la suite.

Reflets : Dans cette création d'un espace de débat local, j'aimerais entendre votre opinion sur le rôle que les femmes ont pris et prennent actuellement.

C.Andrew : Il s'agit de mouvements complexes, parce que les femmes jouent un très grand rôle au niveau local dans la création des services, dans ce que j'appellerais la création des communautés, de tout ce qui se fait dans l'intérêt commun. Cela avec tous les pièges que comporte le bénévolat.Je pense que les femmes sont au coeur de tout ça. Mais cet espace de débat est plus compliqué pour les femmes parce que la parole a souvent été associée, dans la forme formelle ou officielle, aux espaces publics et aux hommes. Les femmes se sont beaucoup parlé, mais n'ont pas eu la parole. Même si le nombre de femmes dans mes cours augmente, j'observe encore ce que j'appellerais la parole appropriée, davantage portée par les hommes. Ceux-ci sont plus habitués de présenter leur position comme chose normale sans devoir tenir compte de la position de l'autre.

Les femmes prennent donc beaucoup de place au niveau local, dans le bénévolat. Mais il y a encore ces passages, parfois difficiles, de la formalisation de ces espaces-là, même dans nos sociétés. Et c'est peutêtre un bon exemple de la complexité de la diversité sociale.

Un autre exemple me vient à l'esprit :il faut être vigilant pour ne pas imposer nos standards de non-sexisme à des sociétés qui gèrent différemment les rapports sociaux de sexe. Mais il faut en même temps envisager la possibilité de sociétés qui gèrent ces rapports d'une façon que nous jugerions sexiste. Ces questions deviennent très compliquées dans notre société : il faut être ouvert à la différence culturelle, mais il faut aussi penser à certaines valeurs qu'on veut maintenir et promouvoir. Certes, il faut faire attention de ne 
pas voir toutes les civilisations avec parti pris mais, en même temps, il faut garder l'égalité comme préoccupation centrale.

Reflets: Au cours des vingt, vingt-cinq dernières années, les femmes ont eu un apport important pour démonter comment la violence affecte à peu près toutes les couches de la société. Qu'est-ce qui se passe actuellement avec la dévolution de l'État, puisqu'il donne moins aux organismes de défense des droits des femmes, par exemple? Les gouvernements, là aussi, se déresponsabilisent, n'est-ce pas?

C.Andrew: Les femmes ont clairement fait un énorme travail afin de rendre visibles, publiques et exprimées de toutes sortes de façons les incidences de la violence. Par exemple, on constate davantage qu'il y a des liens entre les femmes sans-abri et le phénomène de la violence. Le nombre de sans-abri augmente, et on voit comment, dans le cas des femmes, la déstabilisation provoquée par la violence les affecte encore plus durement.

Reflets: Dans la conjoncture actuelle, voyez-vous des particularités à l'analyse que font les femmes des problèmes sociaux en Ontario français?

C.Andrew: Une des dimensions les plus intéressantes, c'est l'impact sur les problèmes sociaux du quasi-retrait du discours des droits. Autour de la loi 8 , il y avait quand même une certaine articulation des francophones comme des " ayants droit ». Je trouve qu'une des choses qui a presque été balayée par les porte-parole de Harris, c'est cette question des droits. On revient constamment sur la question des privilèges, qui sont évidement aléatoires. Et c'est un des aspects qui rend si intéressant l'exemple de l'hôpital Monfort : il pose la question de la place des droits dans les discours. Paradoxalement, les gens les plus démunis ont beaucoup perdu sur le plan des possibilités, tandis que, dans un certain sens, les privilégiés de la société croient avoir des droits. Le discours sur les droits des démunis, des défavorisés, est presque disparu, et cela influence beaucoup l'Ontario français, parce que ce discours était au coeur de l'évolution de la francophonie dans un contexte minoritaire. Il faut une conscience des droits des personnes pour, par exemple, revendiquer des services en français.

Vouloir des services en français, comme on le fait à l'hôpital Montfort, constitue donc un symbole et un exemple en quelque 
sorte de cette conscience du droit d'être servi dans sa langue. Pour l'Ontario français, la perte de ce répertoire discursif des droits est déplorable.

Reflets : On parlait, tout à l'heure, des droits individuels des gens qui en veulent davantage, tandis que les droits collectifs et les droits culturels sont menacés. La priorité est accordée aux droits économiques par rapport aux droits sociaux. Vous constatez donc que les droits sociaux sont en perte de vitesse?

C.Andrew: Je ne dis pas qu'ils sont entièrement disparus. J'estime que cette stratégie de revendication n'a plus la même importance. Cela pose un vrai problème aux francophones qui doivent maintenant demander gentiment des droits, ne pouvant plus faire appel à ce genre de discours de droit, «d'ayants droit ». En même temps, on attend la prochaine décision de la cour, au sujet de Montfort, parce que c'est intéressant de recourir au tribunal. Cette stratégie ajoute une dimension autre au problème et le situe ailleurs que sur le bureau de Mike Harris. La cour n'a pas nécessairement les mêmes critères ni la même analyse que le premier ministre de l'Ontario. Et les décisions des juges peuvent parfois surprendre.

Reflets : Dernière question : on sait que vous êtes engagée dans le gouvernement local.Vous misez sur le local pour reprendre en main des responsabilités en vue de résoudre des problèmes sociaux. Comment un gouvernement local dynamique peut-il contribuer à redresser les problèmes sociaux globaux actuels?

C.Andrew: Une question intéressante! Ce serait quasiment plus facile de demander pourquoi il ne le peut pas. Ce qui nous amène aux problèmes d'absence de ressources au niveau local.L'impôt foncier n'est pas une bonne base pour financer des politiques progressistes. Donc, là, il y a un problème. Ensuite, les municipalités ont établi une tradition, surtout au cours du vingtième siècle, de vouloir restreindre leur vision et leurs responsabilités plutôt que de les élargir. Une sorte de mentalité qui cherche à gérer les choses, plutôt que de se centrer sur les personnes. Mais, ayant dit ceci, on sait que, présentement, le gouvernement fédéral et le gouvernement provincial veulent décentraliser. Sans trop se laisser prendre dans la rhétorique de la possibilité de partenariats entre 
les communautés et le gouvernement local,je pense qu'il y a des traditions qui font en sorte que les gouvernements locaux sont plus prêts à travailler sur un pied d'égalité avec des membres de la communauté et de le faire avec respect, avec un certain sens du potentiel du communautaire. On doit être capable d'imaginer et de voir les interrelations possibles entre les différents services offerts. C'est difficile, pour un gouvernement qui siège à Toronto d'imaginer comment, par exemple, pourrait se faire à Ottawa la coordination entre la personne sans-abri et les services de counselling. Mais c'est concevable à un nouveau local de rassembler les gens qui travaillent sur ces problèmes et de tenter d'harmoniser les services et les problèmes. Je pense que la taille du local présente un potentiel.Évidemment, cela peut être utilisé aussi d'une façon répressive. Les gouvernements locaux ont souvent été les premiers à condamner les pauvres plutôt que la pauvreté et pensent encore souvent à des stratégies pour faire en sorte que ces "méchants pauvres " quittent leur municipalité.

Il y a sans doute énormément de raisons qui incitent le gouvernement provincial à vouloir décentraliser les services. Il pense que le local sera plus catégorique, plus radical. Ces possibilités-là dépendent beaucoup de l'imagination des élus et de la qualité des fonctionnaires. Je pense qu'il y a des gens qui ont la capacité d'être proactifs... Donc là, je me prononce comme étant très optimiste au niveau du local, et je pense qu'il faut faire attention de pas trop l'être. Par contre, on peut aussi dire que puisqu'il y aura évidemment encore de la décentralisation, c'est à nous d'explorer au maximum les possibilités progressistes de ce processus. Reflets: Nous vous remercions, Madame Andrew. 